\title{
Molybdenum oxide nanocolloids prepared by an external field-assisted laser ablation in water
}

\author{
Salvatore Spadaro ${ }^{l^{*}}$, Martina Bonsignore $^{{ }^{*}}$, Enza Fazio $^{l}$, Francesco Cimino $^{2}$, Antonio Speciale ${ }^{2}$, \\ Domenico Trombetta ${ }^{2}$, Francesco Barreca ${ }^{l}$, Antonina Saija ${ }^{2}$, Fortunato Neri ${ }^{l}$ \\ ${ }^{1}$ Dip. di Scienze Matematiche e Informatiche, Scienze Fisiche e Scienze della Terra (MIFT),Università di Messina, 98166 \\ Messina, Italy \\ ${ }^{2}$ Dip. di Scienze Chimiche, Biologiche, Farmaceutiche ed Ambientali, Università di Messina, Italia
}

\begin{abstract}
The synthesis of extremely stable molybdenum oxide nanocolloids by pulsed laser ablation was studied. This green technique ensures the formation of contaminant-free nanostructures and the absence of by-products. A focused picosecond pulsed laser beam was used to ablate a solid molybdenum target immersed in deionized water. Molybdenum oxide nearly spherical nanoparticles with dimensions within few nanometers $(20-100 \mathrm{~nm})$ are synthesized when the ablation processes were carried out, in water, at room temperature and $80^{\circ} \mathrm{C}$. The application of an external electric field during the ablation process induces a nanostructures reorganization, as indicated by ScanningTransmission Electron Microscopy images analysis. The ablation products were also characterized by some spectroscopic techniques: conventional UV-vis optical absorption, atomic absorption, dynamic light scattering, micro-Raman and X-ray photoelectron spectroscopies. Finally, NIH/3T3 mouse fibroblasts were used to evaluate cell viability by the sulforhodamine B assay
\end{abstract}

\section{Introduction}

Over the last decade, laser ablation in liquid (PLAL) is gradually becoming an irreplaceable technique to synthesize metal oxide nanostructures which represent a new type of analytical tools for biotechnology and life science, including diagnosis and therapy of human diseases [1]. Metal oxide nanostructures can act as nanoprobes for biomedical imaging, drug delivery carriers, or as therapeutic agents by themselves. Moreover, they have shown to be excellent antioxidants in vitro and in vivo models, even if their toxicity emerges in some conditions. This is determined by the different physico-chemical properties of metal oxide nanostructures synthesized using mainly chemical approaches and by the testing conditions (i.e. the experimental environment or the kind of cells). For example, Mo exists in three oxidation states and thus can readily participate in redox reactions. Mo oxide nanostructures showed to be effective against cytotoxicity and oxidative stress induced by $\mathrm{H}_{2} \mathrm{O}_{2}$ in human breast MCF-7 and fibrosarcoma HT-1080 cells [2]. On the other hand, Mo oxide nanostructures exhibit excellent antimicrobial activity against Candida albicans and Aspergillus niger and potent cytotoxicity against lung and breast cancer cells (MCF-7 and HEP G2 cell lines) [3].

It is well known that nanosecond, picoseconds and femtosecond PLAL influences the active species differently in plasma, cavitation bubbles and droplets formation which, in turn, determines different nanocolloids characteristics, especially in terms of size and size distribution. PLAL is an excellent preparation method with respect to chemical approach such as sol-gel techniques because of the possibility to prepare high pure ligand-free nanoparticles in a liquid phase ready for manipulation or processing, like incorporation into different matrices or functionalization with biomolecules [4-6]. Unfortunately, the low content of nanoparticles obtained by laser ablation have limited the application of this method as a widely used or even industrial technique. Studies of PLAL have led to the development of various strategies in order to increase nanoparticle productivity (i.e. the amount of nanoparticles fabricated per unit of time). Recently, some efficient approaches for nanostructure assembly have been presented such as electrochemically assisted PLAL, laser writing of nanopatterns in liquids, temperature, magnetic and electric-field PLAL [7]. Laser ablation in liquids with an externally applied electric field extends the traditional laser ablation in liquid approach, allowing a better control of the morphology, size, chemical composition and structure of the oxide nanostructures as well as an increase of the nanoparticles concentration [8]. Firstly, the electric field is believed to transport the charged particles produced in the plasma plume at the electrodes, in a process that resembles electrophoresis [9]. On the other hand, the electric field allows an efficient removal of the bubbles formed both in the volume and on the target. The

* Corresponding author: salspadaro@unime.it (S. Spadaro); martinabonsi@virgilio.it (M. Bonsignore) 
delivery of laser pulses onto the target is more effective and so the ablation rate in liquid results to be higher by a factor of 3 (generally, about $300 \mathrm{mg} / 30 \mathrm{~min}$ ) compared to ablation without electric field (about $100 \mathrm{mg} / 30 \mathrm{~min}$ ).

Here, we report about the synthesis of water nanocolloids of molybdenum oxide by ablating in water a molybdenum target, using a focused picosecond pulsed laser beam. The effect of an external electric field, applied during the ablation process, was also exploited in order to check for modifications of the nanoparticles surface morphology and chemical bonding configurations. Hence, in view of potential biological applications, the molybdenum oxide nanoparticles cytotoxicity towards the fibroblast cell line NIH/3T3 has been investigated.

\section{Experimental}

Pulsed laser ablation of high purity (99.9\%) molybdenum solid target in deionized water $\left(\mathrm{H}_{2} \mathrm{O}\right)$ was carried out. The second harmonic $(532 \mathrm{~nm})$ of a laser operating at $100 \mathrm{KHz}$ repetition rate with a pulse width of 6-8 picoseconds was used. The target was irradiated with a typical laser power of $2.5 \mathrm{~W}$ and an irradiation time of $30 \mathrm{~min}$. The laser beam was focused to a spot of about $75 \mu \mathrm{m}$ in diameter on the surface of the target with a galvanometric scanner having a telecentric objective with a focal length of $163 \mathrm{~mm}$. During the ablation process two $\mathrm{Pt}$ electrodes were immersed in the liquid in such a way to face their basal planes (see Fig. 1). The two electrodes were polarized by applying a DC potential of $100 \mathrm{~V}$ (the estimated electric field is $20 \mathrm{~V} / \mathrm{cm}$ taking into account the electrode distance of $5 \mathrm{~cm}$ ) and, between the electrodes, the plume was left to develop during the ablation. The experiments were performed at different water temperature (from room temperature (RT) up to $80{ }^{\circ} \mathrm{C}$ ) and its values were monitored both before and after the ablation. During the overall process only a moderate increase was found (less than $10^{\circ} \mathrm{C}$ in $30 \mathrm{~min}$ ), compatible with the thermal energy release due to the ablation phenomena.

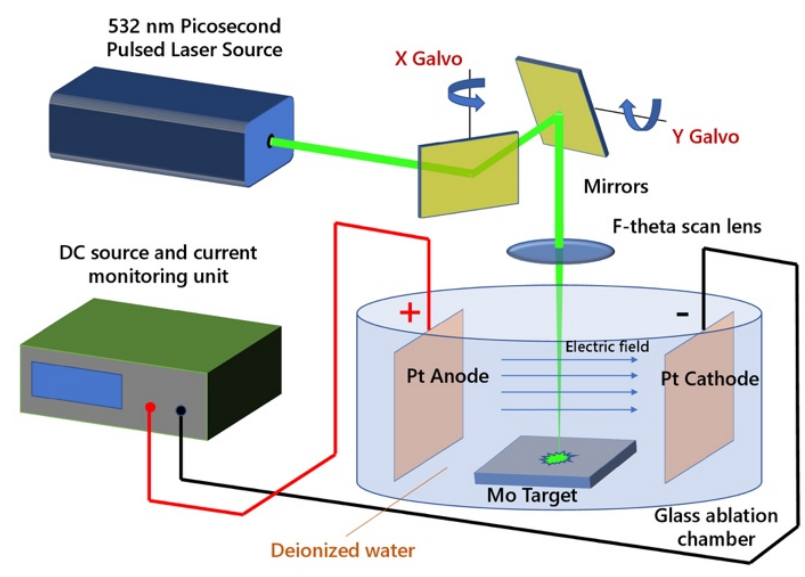

Fig. 1. Scheme of external field-assisted laser ablation setup.

In order to characterize the produced molybdenum oxide nanostructured materials by X-ray photoelectron and micro-Raman spectroscopies, the colloids were transferred on ultra-flat $\mathrm{CaF}_{2}$ and silicon substrates by an ultrasonic spray-casting deposition method. The thickness of the films, measured by a conventional Tencor AlphaStep 500 profilometer, is about $300 \mathrm{~nm}$. The optical transmission of the molybdenum oxide colloids was analyzed by means of a Perkin-Elmer Lambda 750 UVvis spectrometer in the $190-900 \mathrm{~nm}$ range, using quartz cells. Colloids zeta potential was measured with the laser Doppler method, based on the principle of electrophoretic mobility under an electric field, using the Horiba NanoParticle Analyzer SZ-100. A fraction of the colloids was deposited on nickel grids to carry out Scanning Transmission Electron Microscopy (STEM) characterization. STEM images were taken by a scanning electron microscope (ZEISS; model Merlin Gemini 2) operating at an accelerating voltage of $30 \mathrm{kV}$ and at a working distance of $4 \mathrm{~mm}$. The micro-Raman responses of the materials were investigated after the deposition of some drop of the water colloids on a $\mathrm{CaF}_{2}$ substrates. Raman spectra were excited by the $532 \mathrm{~nm}$ radiation of a $30 \mathrm{~mW}$ diode laser, for an integration time of $20 \mathrm{sec}$. The backscattered radiation, collected by an Olympus BX 40 microscope optics using a 100X objective lens, was analyzed by an XploRA $600 \mathrm{~cm}^{-1}$ monochromator equipped with a Peltier CCD sensor. X-ray photoelectron spectroscopy (XPS) spectra were acquired using a KAlpha system from Thermo Scientific, equipped with a monochromatic Al-Ka source $(1486.6 \mathrm{eV})$ and operating in constant analyzer energy (CAE) mode with a pass energy of $20 \mathrm{eV}$ for high resolution spectra and a spot size of $400 \mu \mathrm{m}$. Molybdenum content in colloids was determined by graphite furnace atomic absorption spectroscopy (GF-AAS) using a Varian 220/Zeeman atomic absorption spectrometer (Mulgrade, Victoria, Australia), equipped with a single-element hollow cathode lamp and a Varian PSD autosampler. For the analysis, samples were diluted 1:5000 (v:v) with $0.2 \%$ $\mathrm{HNO}_{3}$ (AAS grade). The quantification of molybdenum was carried out using the external standard method; the reported data are the average of three determinations. The analytical method was validated according to the $\mathrm{ICH}$ guidelines (International Council for Harmonization of Technical Requirements for Pharmaceuticals for Human Use, 2005). The linearity was $>0.999$, the precision (expressed as relative standard deviation) 0.552 , and the detection limit $5.0 \mathrm{ng} / \mathrm{ml}$. The biocompatibility of the colloids on NIH/3T3 cells was investigated using sulforhodamine B (SRB; a dye binding to cellular proteins) assay, as described by Vichai et al. [10] with some modifications. The fibroblast cell line NIH/3T3 was obtained from the American Type Culture Collection (Rockville, MD, USA), and cultured in Dulbecco's modified essential medium (DMEM) supplemented with $10 \%$ fetal bovine serum (FBS), $4 \mathrm{mM}$ L-glutamine, streptomycin and penicillin. Cells were maintained in an incubator with humidified atmosphere containing $5 \% \mathrm{CO}_{2}$ at $37^{\circ} \mathrm{C}$. For the SRB assay, cells were plated in $24-$ wells cell plates at an initial density of $6.5 \times 10^{4}$ cells/well. After $24 \mathrm{~h}$, semi-confluent monolayers were treated for $24 \mathrm{~h}$ with the colloid solutions added to the cell culture medium; control cells were exposed to the same volumes of the vehicle alone (water). Then, cells were fixed using $10 \%$ trichloroacetic acid for $1 \mathrm{~h}$ at $4^{\circ} \mathrm{C}$. After fixation, 
cells were washed twice with water and incubated with $\mathrm{SRB}(0.4 \% \mathrm{w} / \mathrm{v}$ in $1 \%$ acetic acid) for $30 \mathrm{~min}$ at $\mathrm{RT}$, followed by four washes with $1 \%$ acetic acid. The bound dye was solubilized in $1 \mathrm{~mL}$ of $10 \mathrm{mM}$ Tris base solution and the absorbance was measured at $565 \mathrm{~nm}$. Cell viability results are expressed as percentage of viable cells in treated samples with respect to control cells. All the experiments were performed in triplicate and repeated three times. Results are expressed as mean $\pm \mathrm{SD}$ from three experiments and statistically analyzed by a one-way ANOVA test, followed by Tukey's HSD, using the statistical software ezANOVA (http://www.cabiatl.com/ mricro/ezanova/). Differences in groups and treatments were considered significant for $\mathrm{P}<0.05$.

\section{Results and discussion}

The main processes involved in a PLAL process are: generation, transformation, and condensation of plasma plume. Nanoparticles formation is due to competition of these several processes which, in turn, affect nanoparticles properties. We expected that the high repetition rate of ps lasers is advantageous to enhance the production of nanoparticles while the morphology and composition of PLAL-generated nanomaterials can be readily controlled by changing the external environment. Specifically, we expected that the electric field assisted laser ablation leads to the generation of nanoparticles with a narrow size and a well defined distribution. For this purpose, samples were analyzed using different techniques such as STEM, XPS and optical conventional techniques. In Fig. 2 are shown electron microscopy images of the colloids prepared in water at RT and $\mathrm{T}=80^{\circ} \mathrm{C}$, by applying an external $\mathrm{DC}$ electric field. Moreover, the STEM images of the samples prepared without applying the electric field are shown for comparison. Nanoparticles size and their distribution changes as a function of water temperature and electric field. Oblong nanostructures mixed with spherical shaped ones are obtained applying the DC electric field. Particularly, smaller nanoparticles characterize colloids obtained in hot water $(\mathrm{c}, \mathrm{d})$ respect to the ones obtained at room temperature $(a, b)$. Moreover, for this sample, applying the external electric field, the amount of nanoparticles with size less than $15 \mathrm{~nm}$ increases. The optical characteristics and an exemplificative photo of the colloids are shown in Fig. 3. The optical absorption spectra in the $190-900 \mathrm{~nm}$ are generally not representative of the molybdenum oxide formation, since there is no well-defined absorbance band in this spectral region. However, in presence of the electric field, the broad band at higher wavelength could be indicative of the presence of positively charged structural defects, where electrons can be captured by the oxygen vacancies [11]. On the overall, the optical absorption spectra give indirectly semi-quantitative information about the nanoparticles concentrations.

The highest nanoparticles concentration is obtained at the water temperature of $80^{\circ} \mathrm{C}$ and applying the electric field. Mo content was determined by atomic absorption spectroscopy on the basis of three determinations.
Moreover, the colloids are chemically and morphologically stable as indicated by the negative zeta potential values (see Table 1).

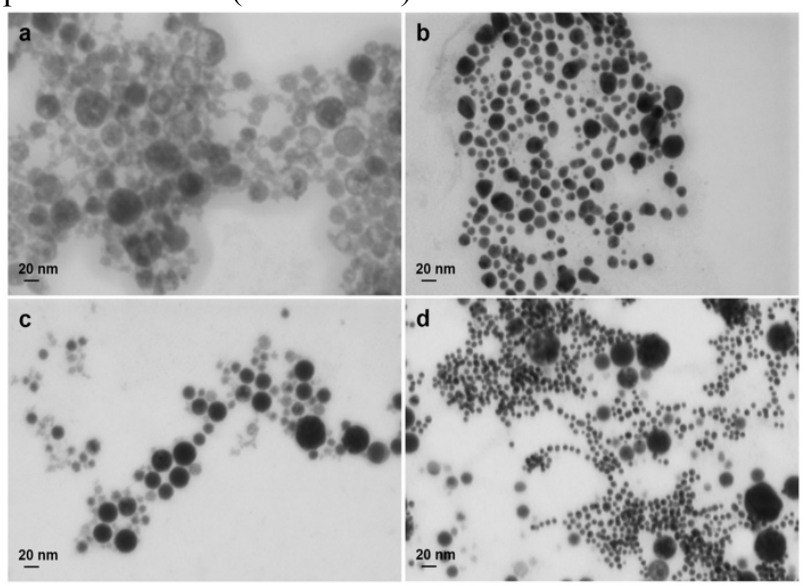

Fig. 2. STEM images of the colloids prepared by an external field-assisted laser ablation in water at (a) RT; (b) $80^{\circ} \mathrm{C}$. For comparison, the images of the colloids prepared without the application of the electric field (c,d) are shown.
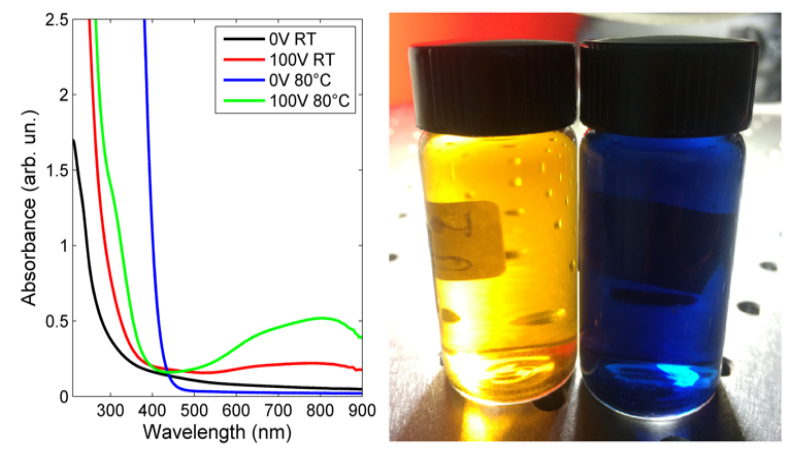

Fig. 3. UV-vis optical absorbance spectra of all samples (left). PLAL colloids exhibit different coloration: yellow without electric field and blue with electric field applied during ablation (right).

Table 1. Zeta potential values and Mo content determined by DLS and atomic spectroscopy, respectively. Data of Mo content are expressed as mean $\pm \mathrm{SD}$ of three determinations.

\begin{tabular}{|c|c|c|}
\hline Sample & $\begin{array}{c}\text { Zeta potential } \\
(\mathbf{m V})\end{array}$ & $\begin{array}{c}\text { Mo content } \\
(\boldsymbol{\mu g} / \mathbf{m l})\end{array}$ \\
\hline Mo_0V_RT & -48.9 & $96.96 \pm 4.8$ \\
\hline Mo_0V_80 ${ }^{\circ} \mathrm{C}$ & -49.4 & $113.43 \pm 1.72$ \\
\hline Mo_100V_RT & -57.5 & $155.75 \pm 3.59$ \\
\hline Mo_100V_80 ${ }^{\circ} \mathrm{C}$ & -42.5 & $171.39 \pm 1.87$ \\
\hline
\end{tabular}

The core level spectra of the samples show the characteristic $\mathrm{Mo}_{3 / 2}$ and $\mathrm{do}_{3 / 2} \mathrm{~d}_{5 / 2}$ doublet caused by spin-orbit coupling. The experimental XPS data were reproduced adopting a fitting model with three contributions ascribed to Mo different oxidation states: $\mathrm{Mo}^{6+}, \mathrm{Mo}^{5+}$ and $\mathrm{Mo}^{4+}$, respectively. The metallic phase is also take into account [12]. 


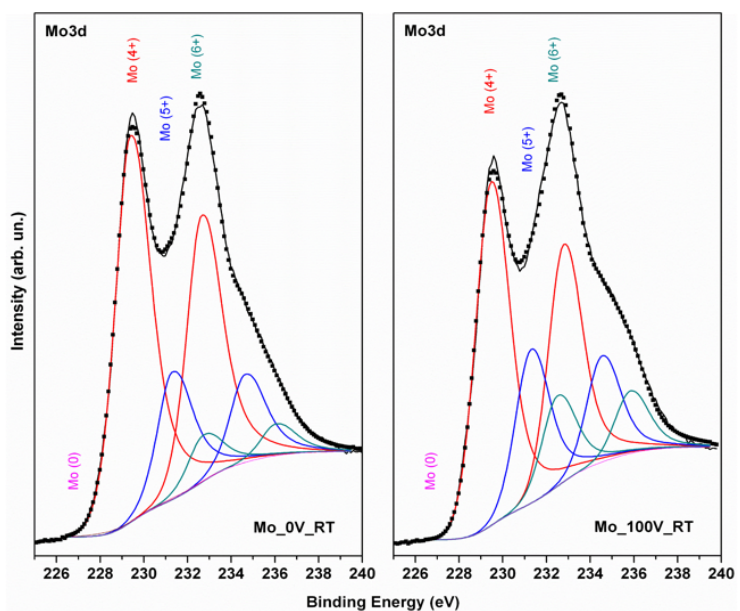

Fig. 4. XPS spectra of the samples prepared at RT but at different electric field (0V and 100V).

In Fig. 4 are shown the XPS high resolution spectra of the samples prepared at RT without and applying the electric voltage (namely, $0 \mathrm{~V}$ and $100 \mathrm{~V}$ ). The application of the external electric field during the ablation process favors the growth of $\mathrm{Mo}^{5+}$ and $\mathrm{Mo}^{6+}$ oxidation phases with respect to the other ones. Similar trend was observed for the samples prepared at the water temperature of $80^{\circ} \mathrm{C}$. The relative abundance of the identified Mo bonding configurations is: $\mathrm{Mo}^{0+}(0.4 \%), \mathrm{Mo}^{4+}(68.7 \%), \mathrm{Mo}^{5+}$ $(22.7 \%)$ and $\mathrm{Mo}^{6+}(8.2 \%)$ for the sample prepared without electric field while $\mathrm{Mo}^{0+}(0 \%), \mathrm{Mo}^{4+}(58.4 \%), \mathrm{Mo}^{5+}$ $(26.2 \%)$ and $\mathrm{Mo}^{6+}(15.4 \%)$ in presence of electric field, respectively. This behaviour may be ascribed to nonequilibrium in nanoparticles charge distribution caused by excessive electrons emitted by cathode, during the plasma expansion. Nanoparticles produced by means of laser ablation in liquids are known to carry negative charge [13]. First, this fact accounts for repulsive forces between nanoparticles and provides their stability against agglomeration, even in absence of surface-active substances. On the other hand, the charge of nanoparticles and therefore potential of their interaction may change if the solution contains a source of charged particles, e.g., electrons. The increase of ion yield and ion charge state by application of an external electric field, as in our case, are most probably due to the trapping of electrons in front of the target surface, which boosts up the electron impact ionization process [14]. All these mechanisms may contribute to define Mo different oxidation states of the investigated samples observed by XPS characterization.

In Fig. 5 are shown the Raman spectra of these samples. The main Raman peaks, belonging to the $\alpha-\mathrm{MoO}_{3}$ orthorhombic structure, centered at about 284, 666, 820 and $995 \mathrm{~cm}^{-1}$ are evident as well as the peaks, associated to the Mo-O stretching mode of the monoclinic $\beta$ phase, located at 776,850 and $900 \mathrm{~cm}^{-1}$. In detail, the contribution at $284 \mathrm{~cm}^{-1}$ represents the bending mode for double bond $(\mathrm{Mo}=\mathrm{O})$ vibration, the peak at $666 \mathrm{~cm}^{-1}$ is assigned to triply coordinated oxygen $\left(\mathrm{Mo}_{3}-\mathrm{O}\right)$ stretching mode, which results from edge-shared oxygen atoms in common to three adjacent octahedral, the band at $820 \mathrm{~cm}^{-}$ 1 is ascribed to doubly coordinated oxygen $\left(\mathrm{MO}_{2}-\mathrm{O}\right)$ stretching mode, which results from corner-sharing oxygen atoms common to two octahedral. Finally, the peak at $995 \mathrm{~cm}^{-1}$ is assigned to terminal oxygen $\left(\mathrm{Mo}^{6+}=\mathrm{O}\right)$ stretching mode, which results from an unshared oxygen, singly coordinated oxygen [15]. The observed vibrational modes confirm the presence of several Mo-O coordinations in good agreement with XPS results.

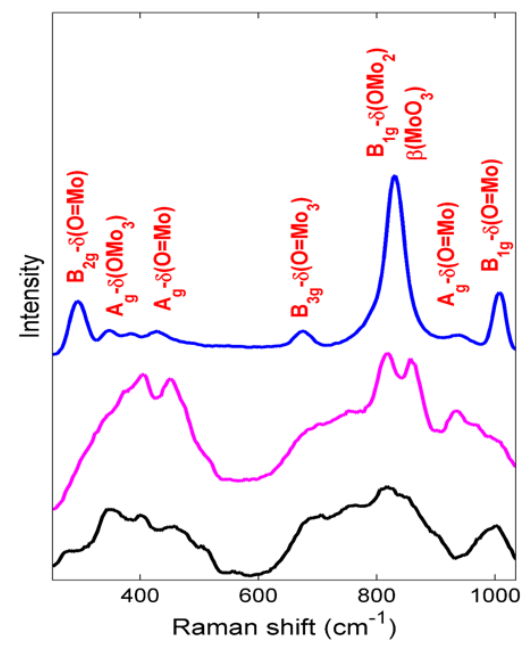

Fig. 5. Raman spectra in different points of the sample prepared at $80^{\circ} \mathrm{C}$ applying the electric field. Similar features were observed for all other samples.

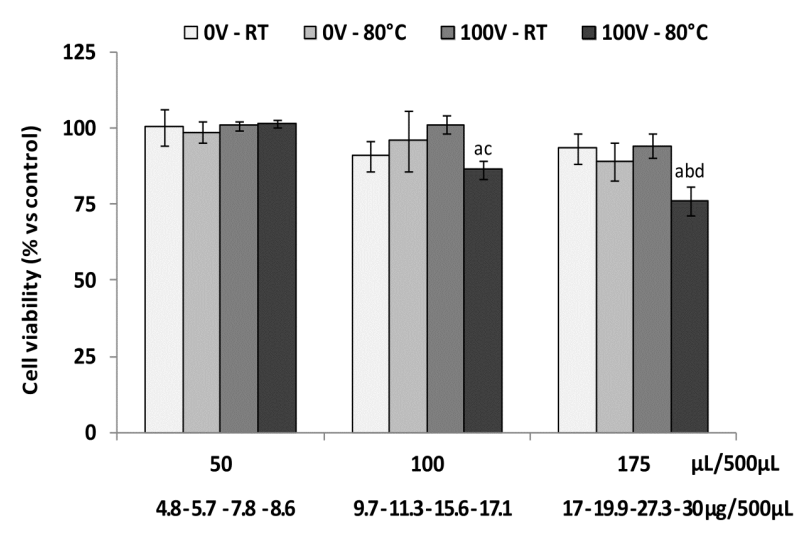

Fig. 6. SRB assay $-\mathrm{NIH} / 3 \mathrm{~T} 3$ cells were treated for $24 \mathrm{~h}$ with the colloid solutions added to the cell culture medium $(500 \mu \mathrm{L}$ total volume). Cultures treated with the same volumes of the vehicle alone (water) were used as controls (CTR). Cell viability data represent percentage of viable cells in treated samples vs CTR. Data are reported as function of the colloid concentration $(\mu \mathrm{L} / 500 \mu \mathrm{L})$ or as Mo concentration $(\mu \mathrm{g} / 500 \mu \mathrm{L}) . a \mathrm{P}<0.05$ vs $50 \mu \mathrm{L}$ of the corresponding colloid; $b \mathrm{P}<0.05$ vs $100 \mu \mathrm{L}$ of the corresponding colloid; $c \mathrm{P}<0.05$ vs $100 \mu \mathrm{L}$ of the colloid $100 \mathrm{~V}$ $\mathrm{RT} ; d \mathrm{P}<0.05$ vs $175 \mu \mathrm{L}$ of the other three colloids.

The surface morphology and the chemical bonding configurations of the synthesized nanoparticles make them an interesting material for biomedical applications. Nevertheless, to become a viable solution, it is mandatory to check their biocompatibility. Thus, the colloid solutions were tested by SRB assay on NIH/3T3 cells. Results showed that only colloids prepared at $80^{\circ} \mathrm{C}$ and applying an external electric field were able to affect, in a 
statistically significant way, cell viability, and this effect was dose-dependent. On the contrary, no cell toxicity was observed for the other colloids tested. Moreover, the size of the molybdenum oxide nanoparticles could play an important role to determine the cell response. Thus, further studies are needed for a deeper understanding of the interaction mechanisms between 3T3 cells and molybdenum oxide nanoparticles by a fine control of the compositional-structural properties of these materials.

\section{Conclusion}

A high efficient and "green" technique based on an external field-assisted laser ablation process is used to prepare water molybdenum oxide nanocolloids. Spherical nanoparticles with dimensions in the $20-100 \mathrm{~nm}$ range are obtained while a nanostructures reorganization is induced by the electric field action. The colloids prepared at $80^{\circ} \mathrm{C}$ and applying an external electric field show dose-dependent cytotoxic effect in NIH/3T3 fibroblasts cells. These results open new prospective for innovative biological applications, such as a potential anticancer complex that has an effect on cellular redox activity.

Authors gratefully acknowledge A.B.A.L. onlus Messina (Italy) (http://www.abalmessina.it) for the use of the XploRA Raman spectrometer.

\section{References}

1. H.S. Tuli, D. Kashyap, S.K. Bedi, P. Kumar, G. Kumar, S.S. Sandhu, Life Sci. 15, 143 (2015).

2. M.J. Akhtar, M. Ahamed, H.A. Alhadlaq, A. Alshamsan, M.A. Majeed Khan, S. A. Alrokayan, J. Coll.and Int. Sci,. 457, 370 (2015).

3. A. Fakhri, P.A. Nejad. J. Photochem. Photobiol B. 159, 211 (2016)

4. E. Fazio, A.M. Mezzasalma, G. Mondio, F. Neri, R. Saija, Appl. Surf. Sci. 272, 30 (2013).

5. E. Fazio, M. Santoro, G. Lentini, D. Franco, S. P. P. Guglielmino, F. Neri, Colloids and Surfaces A: Physicochem. Eng. Aspects 490, 98 (2016).

6. S. Spadaro, M. Santoro, F. Barreca, A. Scala, S. Grimato, F. Neri, E. Fazio, Frontiers of Physics 13(1), 136201 (2018).

7. J. Xiao, P. Liu, C.X. Wang, G.W. Yang, Progress in Materials Science, 87, 140-220 (2017).

8. Y. Li, O. R. Musaev, J-M- Wrobel, M.B. Kruger, J. of Laser Applications 28, 022004 (2016).

9. G. Compagnini, M. Sinatra, P. Russo, G. C. Messina, O. Puglisi, S. Scalese, Carbon 50, 2362 (2012).

10. V. Vichai, K. Kirtikara, Nat. Protoc. 3, 1112 (2006).

11. H.H. Afify, S.A. Hassan, A. Abouelsayed, S.E. Demian, H.A. Zayed, Thin Solid Films, 623, 40 (2017).

12. J.-G. Choi, L.T. Thompson, Appl. Surf. Sci. 93, 143 (1996).
13. J.-P. Sylvestre, S. Poulin, A.V. Kabashin, E. Sacher, M. Meunier, J.H.T. Luong, J. Phys. Chem. B 108, 16864 (2004).

14. C. Pfeiffer, C. Rehbock, D. Huhn, C. CarrilloCarrion, D. Jimenez de Aberasturi, V. Merk, S.Barcikowski, W. J. Parak J. R. Soc. Interface 11:20130931, 1-13 (2014).

15. M. Dieterle, G. Mestl, Phys. Chem. Chem. Phys. 4, 822 (2002). 